\title{
Towards Developing a Backing Layer for Proton Exchange Membrane Electrolyzers
}

P. Lettenmeier ${ }^{1}$, S. Kolb ${ }^{1}$, F. Burggraf ${ }^{1}$, A. S. Gago ${ }^{*}, 1$, K. A. Friedrich ${ }^{1,2}$

${ }^{1}$ Institute of Engineering Thermodynamics, German Aerospace Center, Pfaffenwaldring 38-40,

Stuttgart 70569, Germany

${ }^{2}$ Institute of Energy Storage, University of Stuttgart, Keplerstraße 7, Stuttgart 70174, Germany

*Corresponding author: Tel.: +49 711 6862-8090, fax: +49 711 6862-747, e-mail address: aldo.gago@dlr.de (A.S. Gago). 


\section{Keywords:}

Backing layer; MPL; PEM electrolyzer; PEM electrolysis; current collector; titanium 


\begin{abstract}
Current energy policies require the urgent replacement of fossil energy carriers by carbon neutral ones, such as hydrogen. The backing or micro-porous layer plays an important role in the performance of hydrogen proton exchange membrane (PEM) fuel cells reducing contact resistance and improving reactant/product management. Such carbon-based coating cannot be used in PEM electrolysis since it oxidizes to $\mathrm{CO}_{2}$ at high potentials. A functional titanium macro-porous layer (MPL) on the current collectors of a PEM electrolyzer has been developed by thermal spraying. It improves the contact with the catalyst layers by ca. $20 \mathrm{~m} \Omega \mathrm{cm}^{2}$, increasing significantly the efficiency of the device when operating at high current densities.
\end{abstract}

\title{
Glossary
}

\begin{tabular}{|l|l|}
\hline PEM & proton exchange membrane \\
\hline CC & current collector \\
\hline ICR & internal contact resistance \\
\hline PMG & precious metal group \\
\hline CL & catalyst layer \\
\hline GDL & gas diffusion layer (fuel cell) \\
\hline MPL & macro-porous layer \\
\hline VPS & vacuum plasma spraying \\
\hline LR & leakage rate \\
\hline EIS & electrochemical impedance spectroscopy \\
\hline SEM & scanning electron microscopy \\
\hline CPE & constant phase elements \\
\hline R & resistance \\
\hline OER & oxygen evolution reaction \\
\hline Ir & iridium \\
\hline Pt/C & Platinum on carbon \\
\hline Ti & Titanium \\
\hline
\end{tabular}




\section{Introduction}

Hydrogen can be used as a carbon neutral energy carrier either to provide electricity through fuel cells or to store the surplus coming from renewable sources such as solar or wind by means of water electrolysis systems [1]. Proton exchange membrane (PEM) electrolyzers have great technological potential thanks to the high power densities, superior gas quality and superior dynamic operation ranges [2]. Up to now, the current collector (CC) of a PEM electrolyzer consists of a porous structure of titanium such as a sintered disc, foam, felt or expanded mesh [3]. The porosity and pore size of the sintered discs can be adjusted by selecting the appropriate size and shape of the titanium particles [4] but the configurations are quite limited. Foams, felts and expanded meshes overcome these issues [5] and have a lower cost [6]. However, the development of a $\mathrm{CC}$ with optimized microstructure for efficient water/gas bubble management is still a challenge in PEM electrolysis. F. Arbabi et al. used a technique of visualization of air injection into the liquid-saturated porous network of microfluidic chips in order to simulate the transport mechanism of gas bubbles in the $\mathrm{CC}[7,8]$. They found that transport mechanism of air bubbles in the $\mathrm{CC}$ is capillary-dominated even at high current densities. Their results showed that gas saturation at the breakthrough moment is smaller for felts than for the sintered discs.

The uniform and sufficient contact between the $\mathrm{CC}$ and the catalyst layer (CL) reduces the interfacial or internal contact resistance (ICR) and thus the activation overpotential [9]. The ICR can be lowered by using expensive precious metal group (PMG) coatings on the CC [10]. In the case of a PEM fuel cell this problem is solved by introducing a backing or micro-porous layer between the catalytic layer and the carbon CC or GDL [11,12]. It lowers the ohmic losses by keeping the membrane better hydrated improving the adhesion and contact between the various conductive components, such as carbon [11]. In addition, it allows for better transport of the 
various species required for reaction by removing the produced $\mathrm{H}_{2} \mathrm{O}$ through its hydrophobic component. Lastly, the backing layer in a PEM fuel cell buffers regions between the diffusion media and the CL [12]. All the benefits that it offers to the PEM fuel cell, reported in more than 149 publications since 2002 (ISI Web of Knowledge), are hitherto missing in a PEM electrolyzer. Herein we provide an approach for developing a titanium macro-porous layer (MPL) produced vacuum plasma spraying (VPS) on the CC of a PEM electrolyzer.

\section{Experimental}

Porous titanium coatings were deposited by vacuum plasma spraying (VPS) on $5 \times 5 \mathrm{~cm}^{2}$ sintered titanium filters (SIKA-T 10, GKN Sinter Metals, thickness of 1mm). Only the side of the $\mathrm{CC}$ that is in contact with the CL is coated. Grade 1 titanium (grain size $<45 \mu \mathrm{m}$, TLS Technik Spezialpulver) was used as feedstock powder. The chamber pressure of the VPS facility was 50 mbar in order to prevent the presence of $\mathrm{O}_{2}$, which would oxidize titanium into $\mathrm{TiO}_{2}$ at the high temperatures of the plasma. The substrates were pre-heated up to $250{ }^{\circ} \mathrm{C}$ during deposition. Gas flow rates of $\mathrm{Ar}, \mathrm{N}_{2}$ and $\mathrm{H}_{2}$ were carefully chosen to achieve the highest plasma

enthalpy possible (21.27 $\mathrm{MJ} \mathrm{kg}^{-1}$ ). The torch sweep rate was $500 \mathrm{~mm} \mathrm{~s}^{-1}$. Measurements of the leakage rate (LR) parameter and ICR under compaction force were used as rough indicators of the density and electrical properties, respectively. Experimental details are reported elsewhere [13]. Cross-section images of a coated CC were taken with a SEM Zeiss ULTRA plus (secondary electron detection) with charge compensation. The accelerating voltage and working distance were $15 \mathrm{kV}$ and $8.4 \mathrm{~mm}$, respectively.

Electrochemical tests were performed in $25 \mathrm{~cm}^{2}$ single PEM electrolyzer cells using the porous titanium discs as CC with and without MPL. Commercial MEAs (Solvicore E400) with a N115 
membrane, an Ir-based anode and a Pt/C-based cathode, was employed. Electrochemical impedance spectroscopy (EIS) measurements were realized by means of an impedance equipment (Zahner elektrik IM6) and booster (Module PP240) at different current densities in a frequency range of $1 \times 10^{5}$ to $2 \times 10^{-3} \mathrm{~Hz}$. The water was supplied only to the anode side of by means of a peristaltic pump at a flow rate of $1 \mathrm{ml} \mathrm{s}^{-1}$. A DI water resin (630 Laurent, Grandby, QC J2G 8V1, Aldex Chemical Co. LTD) was used to maintain a resistivity below $10 \mathrm{M} \Omega \mathrm{cm}$. The measurements were recorded at a scan rate of $4 \mathrm{~mA} \mathrm{~cm}^{-2} \mathrm{~s}^{-1}, 85^{\circ} \mathrm{C}$ and atmospheric pressure.

\section{Results}

The proof of concept of a MPL with controlled porosity is illustrated in a scheme presented in Fig. 1a. As shown, the MPL should increase the contact surface with the catalyst while improving the water/gas management through a gradient of pore sizes. For this purpose, several layers of titanium were deposited on the CC. The LR was determined. It was found that two layers of titanium produced a LR of $480 \mathrm{mbar} 1 \mathrm{~cm}^{-2} \mathrm{~s}^{-1}$. Two more layers decreased this value down to 112 mbar $1 \mathrm{~cm}^{-2} \mathrm{~s}^{-1}$. Further increase in the number of layers resulted in lower LR, thus higher density of the coating. Fig. 1b shows a cross section SEM image of 8-layers of porous titanium deposited on the surface of the CC by VPS. As it can be observed the layer has a splat structure, is porous, and rough. An estimation of the thickness, rugosity, pore size and porosity was carried out by image analysis. These results along with the LR measurements are summarized in Table 1. One can notice that the increase of the number of layers has practically no effect in the rugosity, which in average is about 0.74 . However, it is possible to make the coating rougher by increasing the torch sweep rate. By depositing layers of titanium on mild steel and corroding the substrate afterwards it is possible to determine more accurately the pore size of 
a freestanding MPL by Hg-adsorption technique. It turned to be between $0,1 \mu \mathrm{m}$ and $10 \mu \mathrm{m}$ with a modal pore size of $1,3 \mu \mathrm{m}$ (Fig S1. Supporting information). The pore structure of the MPL can be controlled up to a certain extent by varying the deposition parameters. The systematic variation of the VPS parameters for producing MPL with desired properties will be reported in a separate study. Only the 2-layer MPL was chosen for subsequent characterization presented in this study.

The method of measuring ICR $v s$. compaction force is useful for characterizing interconnecting elements of a low temperature electrochemical device such as PEM fuel cell or electrolyzer. Fig. 1c shows the interfacial contact resistance of the CC with and without the VPS coated MPL with respect to compaction force. The difference in their ICR is about $20 \mathrm{~m} \Omega \mathrm{cm}^{2}$ at any compaction force. The decrease in this parameter is owned to a higher contact area of the CC with MPL compared to the uncoated one. Consequently, it is expected the drop in the overpotential of a 25 $\mathrm{cm}^{2}$ PEM electrolyzer should be at least $40 \mathrm{mV}$ at nominal operation of $2 \mathrm{~A} \mathrm{~cm}^{-2}$.

The current-potential characteristics of the PEM electrolyzer without and with coated CC with MPL are presented in Fig. 2a. The inset shows the Nyquist plots measured at $0.4 \mathrm{~A} \mathrm{~cm}^{-2}$. One can observe that the electrolyzer with MPL has lower internal ohmic resistance than the one without MPL. EIS measurements were performed at different current densities in order to gain more insight of the effects of the MPL in the performance of the electrolyzer. An equivalent electrical circuit, Fig. 2b, was used for modeling the results. It consists of a resistor $\left(\mathrm{R}_{1}\right)$ in series with other three $\left(\mathrm{R}_{2}-\mathrm{R}_{4}\right)$, each in parallel with three constant phase elements (CPE) with a constant exponent of 0.8. Additionally, an inductive element $\left(\mathrm{L}_{1}\right)$ connected in series was used for the high frequencies. The Nyquist plots with the measured and simulated spectra are presented in Fig. 2c and Fig. 2d for the electrolyzer without and with MPL, respectively. The 
electrolyzer with MPL shows a decrease of the high frequency loop which corresponds to charge transfer resistance of the electric/ionic conductive elements $\left(\mathrm{R}_{2}\right)$ and double layer charge effects in the active zone of the electrode [14-16].

The computed values of $\mathrm{R}_{1}-\mathrm{R}_{4}$ are presented in Fig. 3a-Fig. 3e. Firstly, the resistance associated to the interconnecting elements and membrane $\left(\mathrm{R}_{1}\right)$ of the electrolyzer decreases ca. $20 \mathrm{~m} \Omega \mathrm{cm}^{2}$ when having an MPL, which is in good agreement with the results from the ICR measurements, Fig. 1c. Similar improvement is observed in the charge transfer resistance $\left(R_{2}\right)$ at the interface of the MPL and catalyst layer/ionomer. Secondly, as expected there is no impact in arc related to the kinetic resistance $\left(\mathrm{R}_{3}\right)$ of the oxygen evolution reaction (OER) either with or without MPL, Fig. 3c. The changes of the low frequency loop measured at high current densities, which correlates to mass transport limitation effects [16], does not follow a clear trend as the values of $\mathrm{R}_{4}$ invert at $0.2 \mathrm{~A} \mathrm{~cm}^{-2}$, Fig. 3d. More experimental data is required to fit the model at high current densities.

Lastly, the MPL on the CC has a moderate impact on the performance of the electrolyzer when operating at current densities below $1.2 \mathrm{~A} \mathrm{~cm}^{-2}$. However, at higher current densities, where mass transport effects dominate, the impact of the MPL in performance is even more profound. At $5 \mathrm{~A}$ $\mathrm{cm}^{-2}$ the overpotential of the electrolyzer is reduced by approx. $256 \mathrm{mV}$ which translates in an increase in efficiency of almost 5\%. It is expected that at full load operation, PEM electrolyzers for the power-to-hydrogen application will run at high current densities in order to save capital investment cost. 


\section{Conclusions}

Here we presented the development and characterization of a MPL produced by thermal spraying on the CC of a PEM electrolyzer. Thanks to its morphological properties, it reduces the ICR by ca. $20 \mathrm{~m} \Omega \mathrm{cm}^{2}$ and mass transport limitations especially at high current densities. The use of thermal spraying for coating the CC of PEM electrolyzers has been shown to provide appropriate structures showing potential for further improvement of the MPL. In this context, the titanium layer in contact with the CL should have small porosity for improved water management at high oxygen production, while the outer layer should have large pore size for rapid release of $\mathrm{O}_{2}$ bubbles. The effect for water and gas transport as a result of incorporating hydrophobic or hydrophilic components to thermally sprayed MPL is still an open question. A deep understanding of mass transport phenomena in the $\mathrm{CC}$, with or without MPL, requires more experiments in combination with modeling.

\section{Acknowledgements}

The authors acknowledge the German BMWi for financial support in the project No. 0325440A. The authors are also grateful to Josef Kallo, Günter Roth, Gudrun Steinhilber, and Ina Plock for additional support, spraying the titanium coatings, the preparation of the samples for analysis, and SEM images, respectively.

\section{References}

[1] A. Sternberg, A. Bardow, Energy Environ. Sci. (2015) 389.

[2] M. Carmo, D.L. Fritz, J. Mergel, D. Stolten, Int. J. Hydrogen Energy 38 (2013) 4901.

[3] H. Ito, T. Maeda, A. Nakano, C.M. Hwang, M. Ishida, A. Kato, T. Yoshida, Int. J. Hydrogen Energy 37 (2012) 7418. 
[4] S.A. Grigoriev, K.A. Dzhus, D.G. Bessarabov, P. Millet, Int. J. Hydrogen Energy 39 (2014) 20440.

[5] C.M. Hwang, M. Ishida, H. Ito, T. Maeda, A. Nakano, A. Kato, T. Yoshida, J. Power Sources 202 (2012) 108.

[6] H.G. Kim, L.K. Kwa, W. Han, L.K. Kwac, W. Han, in:, Int. Conf. Power Energy Syst. Lect. Notes Inf. Technol. Vol.13, 2012, pp. 373-379.

[7] F. Arbabi, A. Kalantarian, R. Abouatallah, R. Wang, J.S. Wallace, A. Bazylak, J. Power Sources 258 (2014) 142.

[8] F. Arbabi, A. Kalantarian, R. Abouatallah, R. Wang, J. Wallace, A. Bazylak, ECS Trans. 58 (2013) 907.

[9] H. Ito, T. Maeda, A. Nakano, A. Kato, T. Yoshida, Electrochim. Acta 100 (2013) 242.

[10] H.-Y. Jung, S.-Y. Huang, B.N. Popov, J. Power Sources 195 (2010) 1950.

[11] S. Park, J.-W. Lee, B.N. Popov, J. Power Sources 163 (2006) 357.

[12] A.Z. Weber, J. Newman, J. Electrochem. Soc. 152 (2005) A677.

[13] A.S. Gago, D. Garcia-Sanchez, S.A. Ansar, P. Gazdzicki, N. Wagner, J. Arnold, K.A. Friedrich, ECS Trans. 69 (2015) 223.

[14] O. Antoine, Y. Bultel, R. Durand, J. Electroanal. Chem. 499 (2001) 85.

[15] M. Eikerling, a. a. Kornyshev, J. Electroanal. Chem. 475 (1999) 107.

[16] J.T. Mueller, P.M. Urban, J. Power Sources 75 (1998) 139. 


\section{Tables}

Table 1. Properties and characteristics of the MPL deposited on the sintered titanium CC, estimated from SEM image analysis. The resulting leakage rate (LR) is presented in the last column.

\begin{tabular}{llllll}
\hline $\begin{array}{l}\text { Number of } \\
\text { Ti-layers }\end{array}$ & $\begin{array}{l}\text { Average } \\
\text { thickness } / \mu \mathrm{m}\end{array}$ & $\begin{array}{l}\text { Rugosity / } \\
\text { a. } \mathrm{u} .\end{array}$ & Pore size / $\mu \mathrm{m}$ & Porosity / \% & $\begin{array}{l}\mathrm{LR} \\
/ \mathrm{mbar}^{2} \mathrm{~cm}^{-2} \mathrm{~s}^{-1}\end{array}$ \\
\hline 2 & 13.4 & 0.83 & 1.6 & 9.3 & 480 \\
4 & 30.8 & 0.62 & 1.7 & 12 & 112 \\
6 & 37.8 & 0.8 & 2.4 & 14.6 & 0.086 \\
8 & 62 & 0.7 & 3.7 & 13.2 & 0.014 \\
\hline
\end{tabular}




\section{Figure captions}

Fig. 1. (a) Scheme of a MPL between the CC and catalytic layer of a PEM electrolyzer. The white pores are interconnected. (b) Cross-section SEM image of a thermally sprayed titanium MPL on a CC. (c) ICR vs. compaction force of one-side coated (MPL) and uncoated (without MPL) CC.

Fig. 2. (a) Current-potential characteristics of PEM electrolyzer single cells with and without MPL. The inset shows the Nyquist plots of both cells at $0.4 \mathrm{~A} \mathrm{~cm}^{-2}$. (b) Equivalent circuit for simulating the EIS data measured at different current densities. (c) and (d) Nyquist characteristics of the cell without and with MPL.

Fig. 3. Calculated values of (a) $R_{1}$, (b) $R_{2}$, (c) $R_{3}$ and (d) $R_{4}$ for different current densities. (e) Current-potential and temperature characteristics of a PEM electrolyzer single cell with and without MPL at full performance. 
Fig. 1.
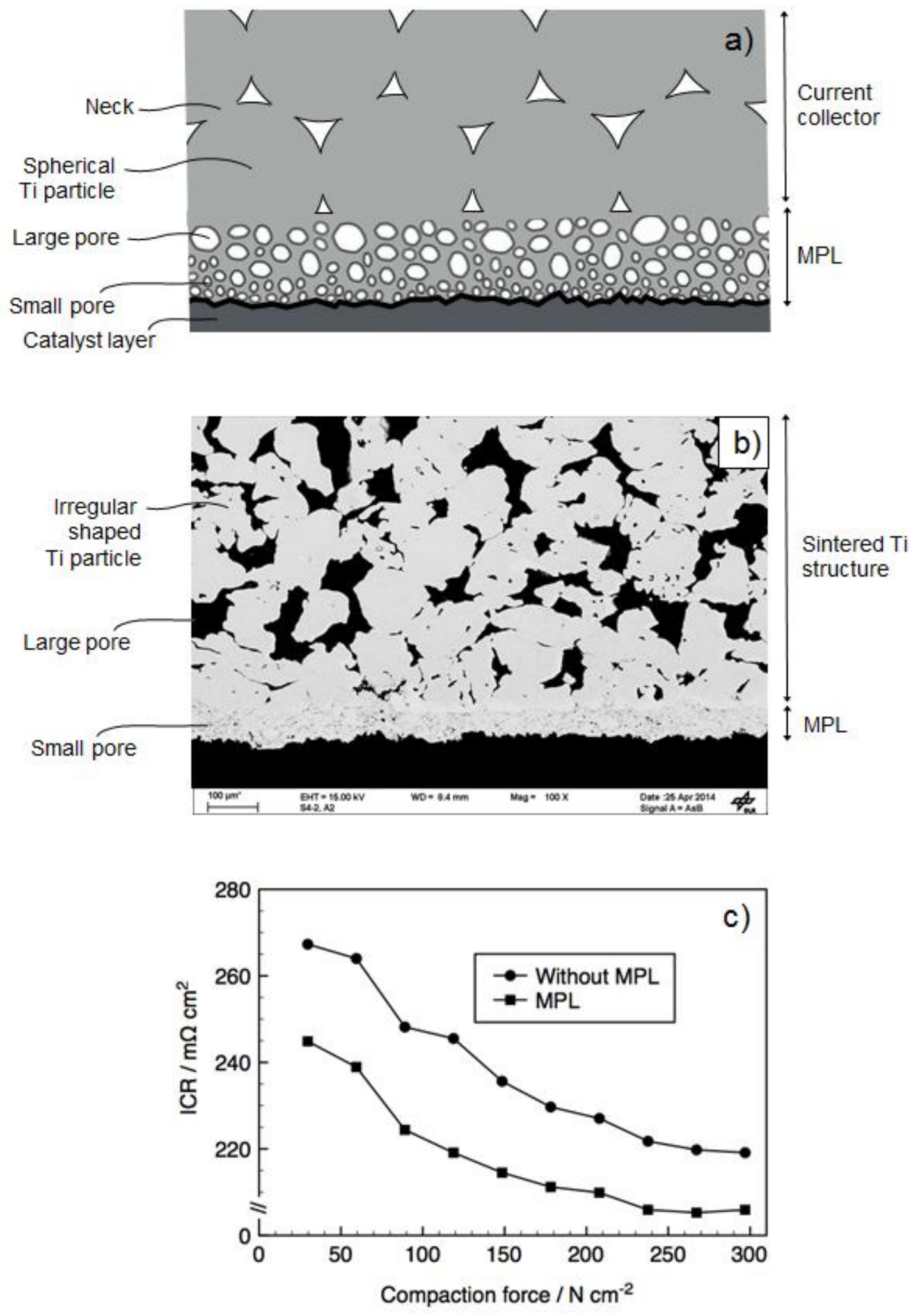
Fig. 2.

a)

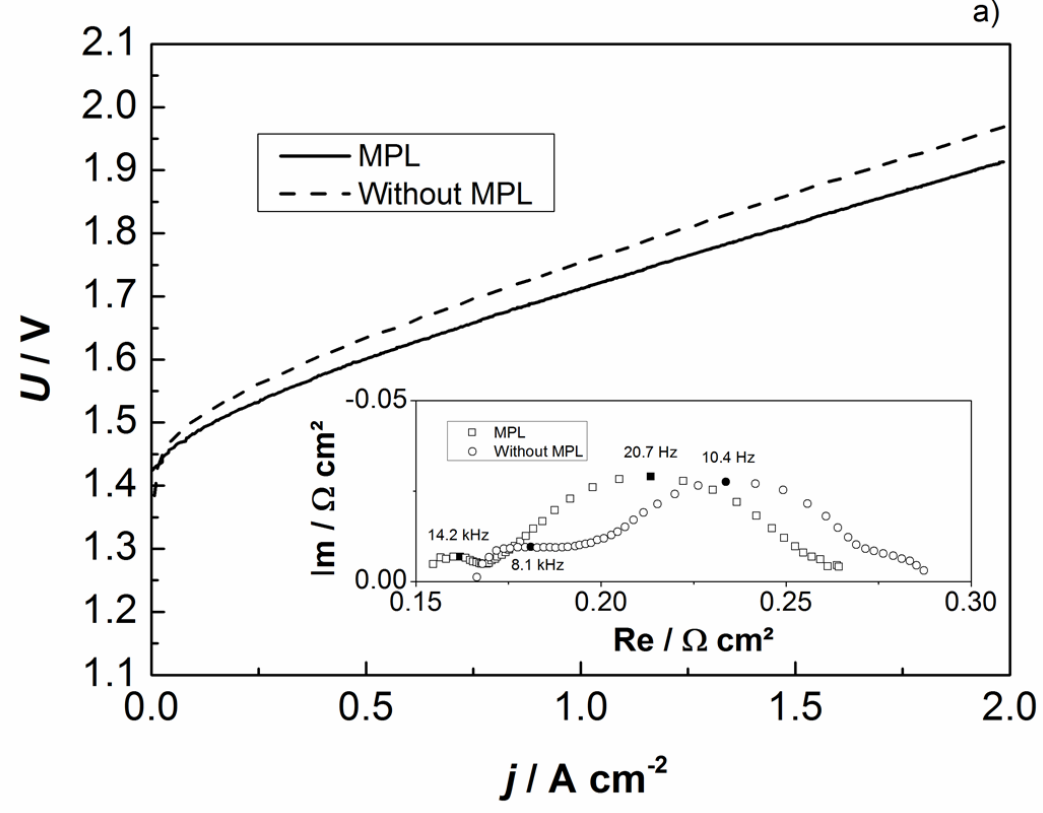

b)

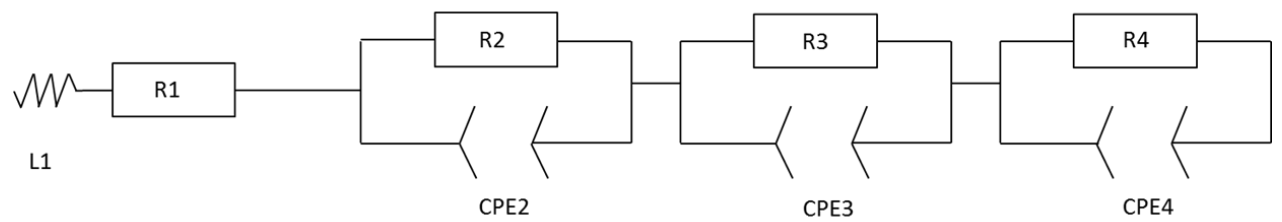

c)

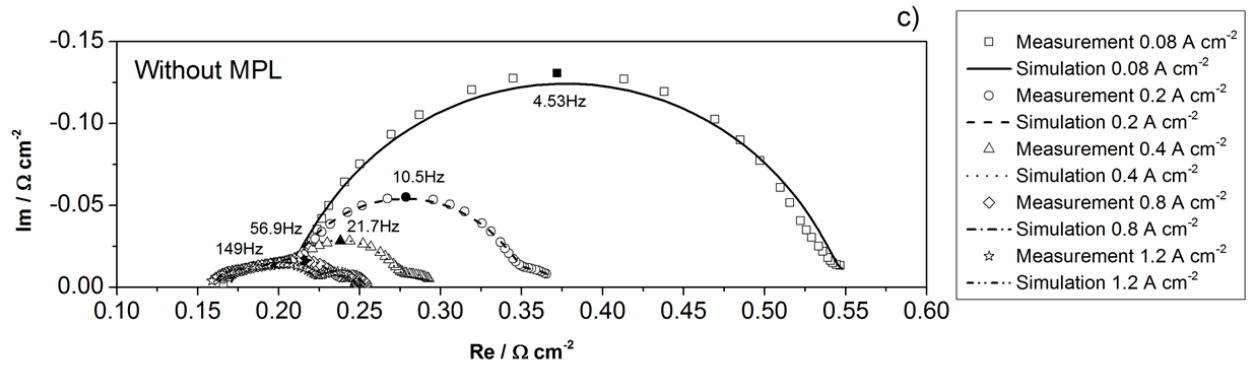

d)

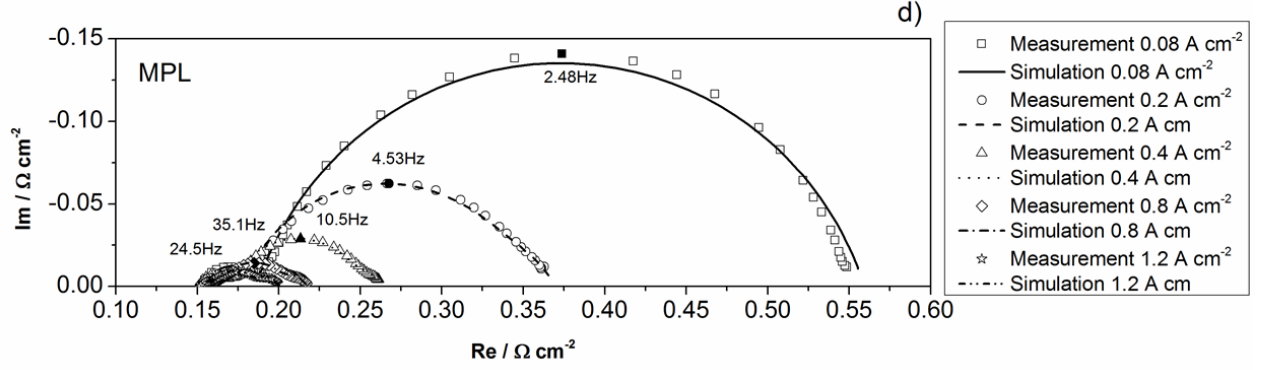


Fig. 3.
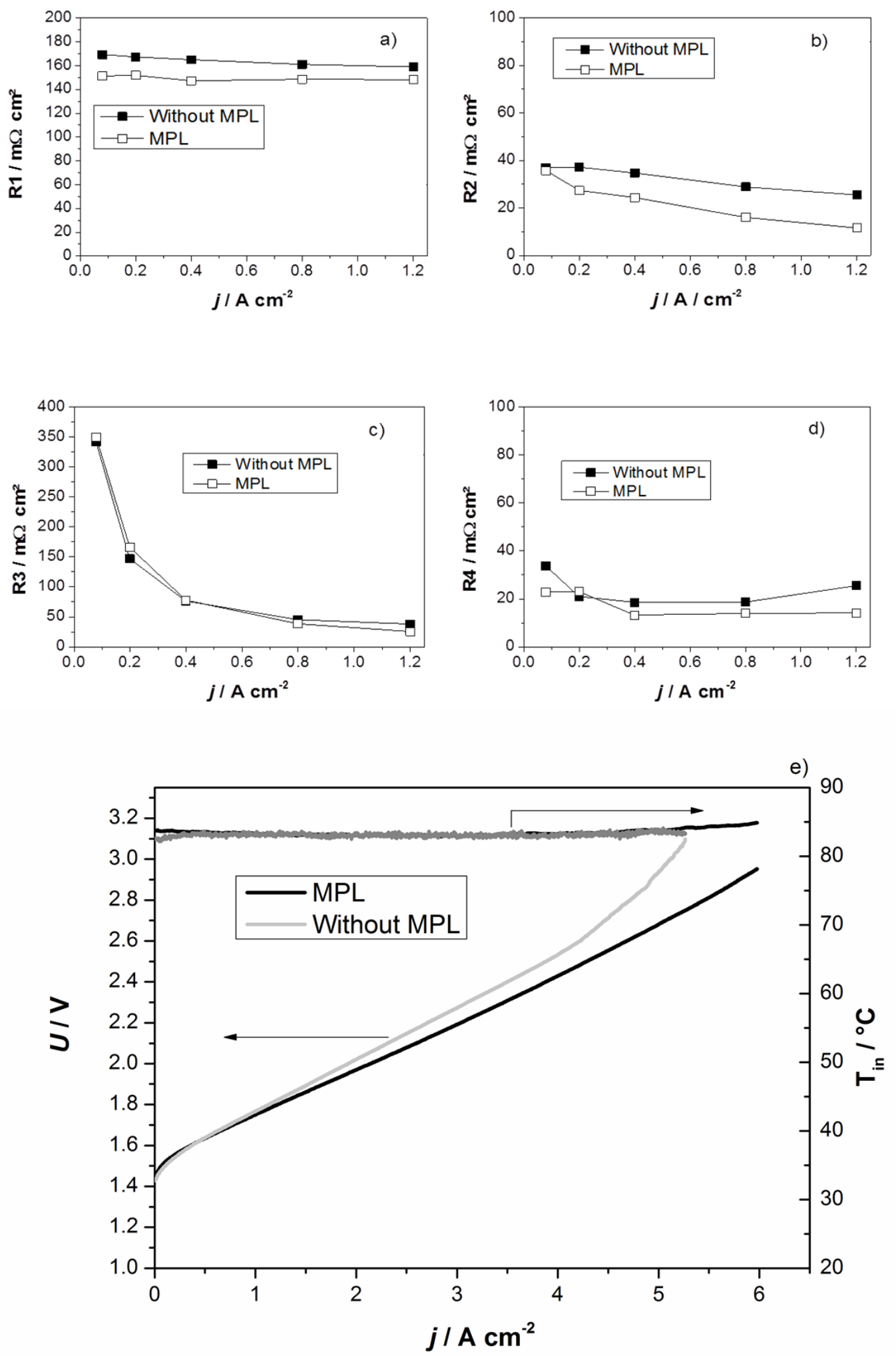

Page 15 